\title{
Fezolinetant findings can fuel future instrumentation inquiries
}

\author{
2020 \\ Carpenter, Janet S. PhD, RN, FAAN \\ School of Nursing, Indiana University, Indianapolis, IN
}

It is well established that menopausal vasomotor symptoms interfere with daily life, affect overall quality of life, and often co-occur with sleep disturbances and other symptoms. It is for this reason that most well-designed efficacy trials include a wide range of patient-reported outcome (PRO) measures. In this month's issue, Santoro et $\mathrm{ll}^{1}$ present PRO data on the efficacy of fezolinetant for hot flash related daily interference, menopausal quality of life, and vasomotor symptom domain scores. Fezolinetant blocks neurokinin B signaling that has been shown to be involved in the etiology of menopausal vasomotor symptoms. ${ }^{2}$ VESTA was a large, phase IIB, randomized controlled trial of seven different Fezolinetant dosing regimens (15, 30, 60, $90 \mathrm{mg}$ BID; 30, 60, $120 \mathrm{mg}$ daily) versus placebo. This carefully designed, rigorous trial had many strengths, including the use of well-validated PRO measures. Fezolinetant, at all doses, was superior to placebo in reducing hot flash interference, improving quality of life, and improving Greene Climacteric Scale vasomotor domain scores.

In addition to fueling new clinical prescribing practices, findings from this study can fuel additional research in the measurement of PROs. Two measures, the Hot Flash-Related Daily Interference Scale and the Menopausal Quality of Life scale have defined minimally important difference values and these were accounted for in the analysis. A minimally important difference is the smallest amount of difference that would lead to changes in patient management. ${ }^{3}$ Changes in Hot Flash-Related Daily Interference Scale

This is the author's manuscript of the work published in final edited form as:

Carpenter, J. S. (2020). Fezolinetant findings can fuel future instrumentation inquiries. Menopause, 27(12), 1347. https://doi.org/10.1097/GME.0000000000001648 
and Menopausal Quality of Life scale scores at 4 and 12 weeks exceeded the defined minimally important differences at all doses as well as for placebo. Because placebo effects are widely documented, perhaps the field should consider defining a new concept, the 'generally anticipated placebo effect'. This concept could be statistically evaluated in the same ways that minimally important differences are established. It could not only help interpret placebo effects, it could set a comparative threshold to use in a priori calculations of statistical power for determining sample sizes.

A third measure, the Greene Climacteric Scale, does not have a defined minimally important difference. The measure has been widely used and it is surprising that a minimally important difference has not previously been published. Perhaps the fezolinetant study and this editorial will motivate a reader to use their own data to fill this glaring gap and move the field forward.

A fourth measure, not previously mentioned in this editorial, was the Leeds Sleep Evaluation Questionnaire. Santoro et $\mathrm{al}^{1}$ indicate that because of issues with operationalizing the assessment, the results were invalid and thus, not reported. The gap in findings created by this problem speak to the difficulties researchers encounter in balancing brevity of PRO assessments with redundancy of using more than one measure of any given concept. A second sleep measure was not included, likely because of concerns about participant burden, but doing so could have provided a backup for the problematic measure. For now, additional research with fezolinetant will be needed to establish its impact on sleep disturbances. 


\section{References}

1. Santoro N, Waldbaum A, Lederman S, et al. Effect of the neurokinin 3 receptor antagonist fezolinetant on patient-reported outcomes in postmenopausal women with vasomotor symptoms: results of a randomized, placebo-controlled, double-blind, dose-ranging study (VESTA). Menopause 2020; 27:13501356.

2. Modi M, Dhillo WS. Neurokinin 3 receptor antagonism: a novel treatment for menopausal hot flushes. Neuroendocrinology 2019; 109:242-248.

3. Guyatt GH, Osoba D, Wu AW, Wyrwich KW, Norman GR. Methods to explain the clinical significance of health status measures. Mayo Clin Proc 2002; 77:371-383. 